\title{
Políticas habitacionales en la Ciudad de San Luis (2011-2015)
}

\section{Leandro Martín Pérez ${ }^{1}$}

\section{Universidad Nacional de San Luis}

\section{Aporte de Estudiante}

Resumen: El objetivo de este texto es describir las políticas habitacionales que se implementaron en el ámbito del Municipio de la Ciudad de San Luis, Provincia de San Luis, entre los años 2011-2015. Tales políticas fueron ejecutadas en el marco de un Macro Plan de Acceso a la Vivienda que comprendió tres principales programas -entre otras acciones complementarias- que son descriptos en este artículo. No solo se mencionan sus características sino también su estado de ejecución hasta el año que comprende este trabajo. Asimismo, dado que se entiende que toda política pública emerge como respuesta a una necesidad social que logra tener relevancia pública, se describen algunos de los motivos que dieron lugar a la implementación de esas acciones estatales. Sin dudas, tales políticas responden a un déficit habitacional -producto del incremento poblacional, entre otros factorespresente en la Ciudad de San Luis que el Macro Plan no logra resolver en su totalidad, aunque constituye un importante paliativo.

Se trata de un estudio exploratorio con enfoque cualitativo en donde se ha acudido al análisis documental y entrevistas semi-estructuradas a funcionarios públicos. Dicho estudio se realizó en el marco de una pasantía educativa en la Municipalidad de San Luis como estudiante de la Lic. en Ciencia Política.

\section{Introducción}

El objetivo de este aporte es describir las políticas habitacionales llevadas a cabo por la Municipalidad de la Ciudad de San Luis en el período 2011-2015 a través del Macro Plan Municipal de Acceso a la Vivienda.

Dados los cambios socioeconómicos producidos en la última década del siglo XX en Latinoamérica, que redundaron en mayores desigualdades sociales, se produjeron dificultades en el acceso a la vivienda: "En materia habitacional esto ha implicado que el déficit mundial de viviendas en los años 90 afecte a 4000 millones de personas, mientras que el déficit de viviendas en América Latina llegó a 35 millones” (Gargantini, 2005, p. 26).

En el caso específico de Argentina, la población urbana ha crecido en mayor proporción que la población total, aunque dicho proceso de urbanización no se ha

\footnotetext{
${ }^{1}$ Estudiante avanzado de la carrera de Abogacía de la Universidad Nacional de San Luis. Licenciado en Ciencia Política (U.N.R.C.). Correo electrónico: martinperezmpe@gmail.com
} 
desarrollado en todas las provincias en un mismo momento. La Provincia de San Luis, tras el Censo Nacional del año 2010 arroja los siguientes datos: entre el año 2001 y 2010 pasó de tener 372.849 habitantes a 456.767 (una tasa de crecimiento anual medio del 22,8\%). Asimismo, en las mediciones hechas por el Instituto Nacional de Estadísticas y Censos (INDEC) en el Departamento Juan Martín de Pueyrredón donde se ubica la Ciudad de San Luis, se puede observar un alto crecimiento desde los años 1990 hasta la actualidad: 1991 (121.004); 2001 (168.771); 2010 (204.019).

Frente al problema habitacional, la Municipalidad responde diseñando y ejecutando políticas públicas estatales tendientes a reducir el déficit. Bajo ese marco, el texto intenta contribuir a evidenciar de forma clara las respuestas ejecutadas por el Estado municipal al problema habitacional que padece la Ciudad de San Luis a través de las políticas públicas enmarcadas en el Macro Plan de Vivienda.

\section{Desarrollo}

Desde que asumió la conducción de la gestión municipal capitalina en el año 2011, se adoptó la decisión de ejecutar una política de vivienda auto gestionada por el propio municipio. A través de múltiples acuerdos con el gobierno nacional en búsqueda de financiamiento, se inició un proceso de planificación estratégica que incluyó la adquisición de terrenos propios, el trazado de planos, llamado a licitación, contratación de empresas constructoras, supervisión de obras, etc., que hasta el año 2015 se presentaba bajo la modalidad de tres tipos de planes de vivienda municipal enmarcados en el Macro Plan:

\subsection{Plan Social}

Es un programa mediante el cual se gestionan y construyen viviendas con fondos tanto municipales como nacionales, con el objetivo de aportar soluciones habitacionales desde la gestión municipal para reducir el déficit habitacional acumulado de la Ciudad de San Luis. Dicho Plan contaba con un total de 186 viviendas adjudicadas más 386 pre adjudicadas.

En este marco, el 15 de julio de 2013 se entregó el primer barrio construido por la Municipalidad de San Luis con administración y fondos propios compuesto por 42 viviendas; constituyó el primer barrio realizado por una gestión municipal en la Provincia de San Luis. El mismo fue entregado con todos los servicios incluidos: agua, cloacas, alumbrado público y pavimento. La inversión total fue de $\$ 15.174 .795$, monto obtenido por acuerdos entre la Municipalidad y el Ministerio de Planificación Federal, Inversión Pública y Servicios.

Por otra parte, también con fondos nacionales, se estaban construyendo 600 viviendas distribuidas en predios del Extremo Sur de la Ciudad (256 viviendas) y en la zona 
oeste en el terreno de Cazorla (156 viviendas). Doscientas de estas casas fueron entregadas en abril de 2014.

\subsection{Plan de Viviendas para Jóvenes 25/35}

Esta segunda política pública, implementada posteriormente al comienzo de ejecución de la primera, tiene por objetivo facilitar el acceso a la vivienda a núcleos familiares y solteros jóvenes que actualmente no pueden obtener un crédito hipotecario para acceder a su primera vivienda, pero que tengan ingresos demostrables para el pago de una cuota por ella.

En una primera etapa, a través de una inversión de $\$ 20.000 .00$ se construyeron 100 casas en un terreno colindante al barrio Néstor Kirchner, ubicado al sur de la ciudad.

A través del sorteo de Lotería Nacional se determinaron los primeros 100 adjudicatarios de un total de 1.200 inscriptos, quienes comenzaron a abonar 72 cuotas de $\$ 2.083$ sin interés. La entrega de estas 100 viviendas se realizó el día 08 de mayo del año 2015. Las mismas tienen una superficie cubierta de 55 metros cuadrados, con dos habitaciones, un baño, una lavandería y una cocina comedor.

\subsection{Plan Mi Primera Vivienda}

Esta tercera política pública es una continuación del plan descripto en el 2.2 con algunas modificaciones en los requisitos.

En el mes de enero del año 2015 se sortearon 100 casas a través de la Lotería Nacional en su versión vespertina de los martes, y se determinó como fecha de entrega el 18 de mayo del mismo año.

Paralelo a estos tres planes, el ejecutivo capitalino firmó el Plan Maestro Neo San Luis para el Desarrollo Estratégico Urbano 2011-2030 con el objetivo de conformar una Unidad Ejecutora para la Urbanización del predio Granja La Amalia, Estación de Trenes y Conectividad del Corredor Vial, que están íntimamente vinculados con el desarrollo y urbanización de la zona oeste de la ciudad donde actualmente se encuentran algunos de los barrios entregados.

Es sumamente necesario entender cuál fue la causa que condujo a la implementación de este conjunto de políticas públicas, y que a su vez es un problema de principal atención por parte del municipio, incluso durante los periodos actuales en los cuales las soluciones al mismo ya se están ejecutando. La Ciudad de San Luis enfrenta en tiempos actuales un grave déficit habitacional. Dentro de los principales insumos de información para la definición de políticas y programas del sector habitacional la conceptualización y medición del déficit de viviendas se torna indispensable. Marta Baima de Borri (1994) establece dos criterios esenciales para describir y cuantificar el déficit de stock habitacional. El primero es el índice 
de "Precariedad" que toma en consideración ciertas deficiencias por carencias constructivas o de servicios según los tipos de viviendas existentes (departamento alquilado, inquilinato, vivienda individual, tipo casa, rancho, vivienda usurpada, etc.). Tal estado de situación es dable de observar en un aglomerado urbano como el de la Ciudad de San Luis. Deteniéndonos en este primer punto, por medio de una entrevista personal (12/03/15) a la Lic. María José Domínguez (Directora de Política Habitacional de la Ciudad de San Luis), se pudieron obtener una serie de datos que nos dan una primera aproximación al problema del que estamos tratando:

La Ciudad de San Luis posee un considerable número de asentamientos en predios fiscales y no fiscales usurpados, incluso dentro de las cuatro principales avenidas. A esto se le suma una alta tasa de alquileres, y también un alto índice de personas que habitan viviendas prestadas momentáneamente. En nuestra Secretaría es impresionante la cantidad de solicitudes de vivienda, con más de 6.000 personas inscriptas. El problema se agudiza debido a la falta de contención y construcción de refugios por parte de la Provincia.

Dicha afirmación se respalda con los datos obtenidos durante las entrevistas realizadas el día lunes 16/03/15 por la funcionaria Domínguez a 18 inscriptos en el Plan de Viviendas Sociales. De un total de 18 casos (100\%) analizados el día mencionado, un 44\% habita una vivienda prestada y cerca de un $28 \%$ usurpa, lo cual refleja más de un $72 \%$ de inscriptos que no tienen asegurada su permanencia en la vivienda que habitan. Si bien esto es una ínfima muestra sobre los 6384 inscriptos, claramente empieza a demostrar una tendencia.

En las visitas realizadas durante la práctica a los domicilios de los inscriptos, se pudo apreciar un gran número de familias que habitan viviendas que si bien presentan rasgos de precariedad (falta de terminaciones, carencia de instalaciones sanitarias, techos que se llueven, problemas de humedad), son pasibles de ser recuperadas mediante obras de refacción o complemento.

El segundo criterio esencial para describir y cuantificar el déficit del stock habitacional es el de "Hacinamiento", consistente en la relación entre el número de personas que habitan una unidad con el número de cuartos disponibles (Baima de Borri, 1994). Se considera nivel de hacinamiento crítico a más de dos personas por cuarto, tal como se pudo apreciar en varias de las visitas hechas a los domicilios de los destinatarios del Plan de Viviendas. Asimismo, los resultados obtenidos a partir de la base de datos de los inscriptos a los tres planes muestran que sobre un total de 6384 casos (100\%) la mayoría de ellos tiene -en 
promedio- más de tres hijos. El problema se profundiza debido a la precaria situación de salud de un alto número de inscriptos, ya que más del $7 \%$ de la totalidad de ellos (439 personas) padece algún problema de discapacidad.

\section{Reflexiones finales}

El Macro Plan de Viviendas ejecutado por el municipio capitalino encuentra su plena justificación en un escenario concreto y palpable de déficit habitacional.

Uno de los aspectos más destacables de las políticas públicas de vivienda impulsadas y ejecutadas por la Municipalidad de San Luis es la dirección que se les dio a las mismas, con un claro sentido social.

Los Estados municipales, provinciales y nacionales del siglo XXI deben modernizarse, esto implica mayor cercanía con el ciudadano, desburocratización de los trámites y flexibilización en la gestión, para lograr así el cumplimiento de las metas y plazos en tiempo y forma. Esto luego naturalmente se traduce en capital político al momento de las elecciones, permitiéndole continuidad a los proyectos en el largo plazo.

\section{Referencias bibliográficas}

De Borri, M. E. (1994). Hábitat: su problemática y propuestas alternativas. Editado en Seminario de SEHAS-ONG sobre Hábitat. Córdoba.

Gargantini, D. (2005). Gestión local del hábitat: experiencias en municipios intermedios. Córdoba: EDUCC Editorial de la Universidad Católica de Córdoba.

\section{Otros documentos consultados}

Instituto Nacional de Estadísticas y Censo. Censo Nacional de Población, Hogares y Viviendas 2010: resultados definitivos. República Argentina. 